\title{
THE BOOSTER FOR THE CANADIAN LIGHT SOURCE
}

\author{
L. Praestegaard ${ }^{1}$, H. Bach ${ }^{1}$, L. O. Dallin ${ }^{2}$, L. Kruse ${ }^{1}$, S. P. Møller ${ }^{3}$, \\ B. R. Nielsen ${ }^{1}$, F. Pérez ${ }^{1}$, and R. M. Silzer ${ }^{2}$ \\ ${ }^{1}$ Danfysik A/S, DK-4040 Jyllinge, Denmark \\ ${ }^{2}$ CLS, University of Saskatchewan, 107 North Road, Saskatoon, Canada S7N 5C6 \\ ${ }^{3}$ Institute for Storage Ring Facilities, University of Aarhus, DK-8000 Aarhus C, Denmark
}

\begin{abstract}
The design of the 2.9-GeV full-energy booster synchrotron for the Canadian Light Source (CLS) at the University of Saskatchewan, Saskatoon, Canada is presented. Special emphasis is put on the beam acceleration provided by two 500-MHz DORIS-type five-cell cavities which are driven by a single $75 \mathrm{~kW}$ klystron. In particular, the effect of beam loading and eddy currents in the dipole magnet vacuum chamber are considered. The booster synchrotron will deliver an electron beam with a beam energy of 1.5-2.9 GeV, a beam current of more than $10 \mathrm{~mA}$, a pulse length of $\sim 137 \mathrm{~ns}$, and a horizontal emittance of $523 \mathrm{~nm}$ at $2.9 \mathrm{GeV}$. The complete booster is designed and built by the company Danfysik A/S based on an initial design by CLS staff.
\end{abstract}

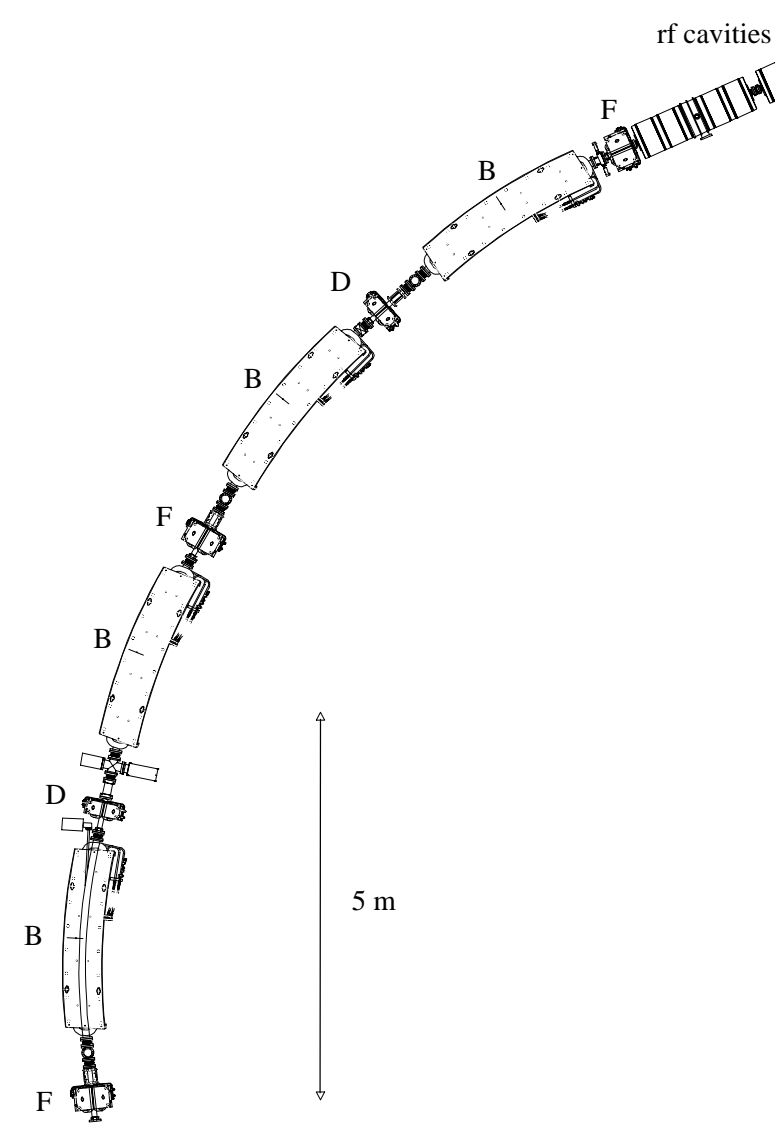

Figure 1: One quarter of the CLS booster synchrotron ( $\mathrm{B}=$ bending magnet, $\mathrm{F}=$ horizontally-focusing quadrupole magnet, and $\mathrm{D}=$ vertically-focusing quadrupole magnet)

\section{INTRODUCTION}

The 2.9-GeV CLS booster synchrotron is the fullenergy electron injector for the Canadian Light Source (CLS) at the University of Saskatchewan, Saskatoon, Canada [1]. After injection of the 250-MeV electron beam from the upgraded Saskatchewan Accelerator Laboratory linac, the booster synchrotron will accelerate a beam current of more than $10 \mathrm{~mA}$ up to a maximum energy of $2.9 \mathrm{GeV}$ and subsequently extract the beam

for injection into the CLS storage ring; a sequence which is repeated with a frequency of $1 \mathrm{~Hz}$. The complete booster synchrotron is designed and constructed by the company Danfysik A/S based on an initial design by CLS [2]. The booster synchrotron will be operational in the beginning of 2002.

Table 1: Main design parameters of the CLS booster synchrotron.

\begin{tabular}{|l|c|}
\hline Injection beam energy & $250 \mathrm{MeV}$ \\
\hline Extraction beam energy & $1.5-2.9 \mathrm{GeV}$ \\
\hline Circumference & $102.528 \mathrm{~m}$ \\
\hline Number of dipole magnets & 20 \\
\hline Dipole field & $0.115-1.333 \mathrm{~T}$ \\
\hline Rf frequency & $500 \mathrm{MHz}$ \\
\hline Residual gas pressure & $<10^{-7} \mathrm{mbar}$ \\
\hline Repetition frequency & $1 \mathrm{~Hz}$ \\
\hline Synchrotron rad. loss per turn & $0.048-863 \mathrm{keV}$ \\
\hline Tunes (hor./ver.) & $5.18 / 2.38$ \\
\hline Chromaticity (hor./ver.) & $-6.87 /-4.03$ \\
\hline Momentum compaction factor & 0.0512 \\
\hline Natural hor. emit. (2900 MeV) & $523 \mathrm{~nm}$ \\
\hline Natural mom. spread (2900 MeV) & $9.2 \cdot 10^{-4}$ \\
\hline Extracted current & $>10 \mathrm{~mA}$ \\
\hline Pulse length of extracted beam & $\sim 137 \mathrm{~ns}$ \\
\hline
\end{tabular}




\section{LATTICE}

One quarter of the CLS booster synchrotron is presented in figure 1 and its main parameters are listed in table 1. The lattice is a 28 -fold super-symmetric FODO lattice in which the required straight sections for injection, $\mathrm{rf}$ cavities, and extraction have been obtained by removing 8 dipole magnets, leaving 20 dipole magnets and only two super-periods in the lattice. This reduces the circumference of the booster synchrotron compared with a genuine FODO lattice. The transverse focusing is provided by a family of horizontally-focusing quadrupole magnets, a family of vertically-focusing quadrupole magnets, and the vertically-focusing end-poles of the rectangular dipole magnets, facilitating horizontal and vertical tunes which can be changed freely in the intervals 5-6 and 2-3, respectively. The resulting lattice functions at the nominal working point $(5.18,2.38)$ are shown in figure 2.

For correction of the closed orbit deviation, produced by magnetic field and alignment errors, 14 horizontal and 8 vertical corrector magnets are distributed uniformly in the lattice. The generation of a large number of closed orbit deviations arising from random errors show that the average horizontal and vertical closed orbit deviation after orbit correction are $0.6 \mathrm{~mm}$ and $1.0 \mathrm{~mm}$, respectively, for 27 beam position monitors for detection of the beam position.

Finally, sextupole magnets are not implemented in the lattice for correction of the natural chromaticities to positive values since the head-tail instability is not considered to cause any problems for a beam current below $\sim 50 \mathrm{~mA}$.

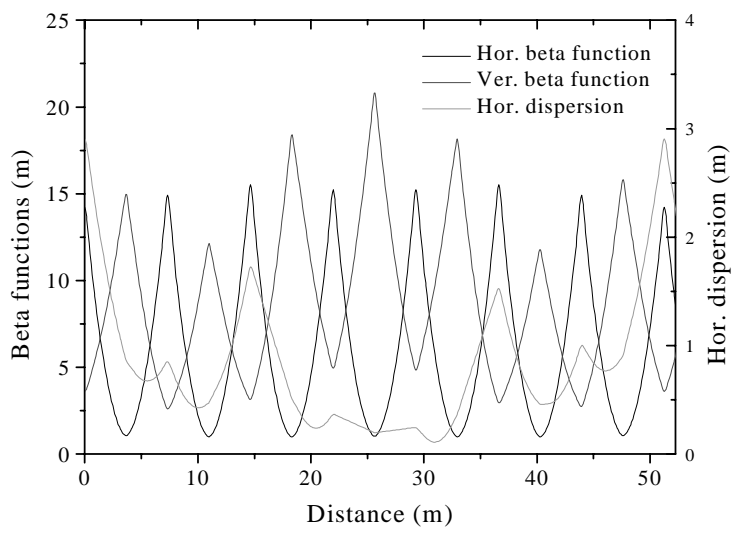

Figure 2: Lattice functions in one super-period of the lattice of the CLS booster synchrotron at the nominal working point $(5.18,2.38)$.

\section{INJECTION}

The 2856-MHz pre-injector linac delivers a $250-\mathrm{MeV}$ electron beam with a maximum bunch charge of $\sim 50 \mathrm{pC}$, a pulse length of $\sim 137 \mathrm{~ns}$, truncated transverse emittances of $300 \mathrm{~nm}$, and a relative momentum spread of 0.0015 . Furthermore, the $2856-\mathrm{MHz}$ pulse train is chopped in $\sim 90^{\circ}$ long $500-\mathrm{MHz}$ bunches in order to enhance the capture efficiency of the injected beam in the booster synchrotron.
At the end of the linac-booster synchrotron transfer line, the linac beam enters a pulsed injection septum magnet which deflects the beam $10.358^{\circ}$ to a final inclination towards the axis of the booster synchrotron of $0.642^{\circ}$. After passage of a vertically-focusing quadrupole magnet, the beam finally intersects the axis of the booster synchrotron $3.533 \mathrm{~m}$ downstream of the septum magnet at which point the beam is deflected on axis by a fast injection kicker magnet. The falltime of the excitation pulse of the kicker magnet is less than $190 \mathrm{~ns}$, ensuring that the beam is not deflected during the second passage of the kicker magnet. Hence, the injection process will inject the whole pulse train of the pre-injector linac on the axis of the booster synchrotron.

\section{BEAM ACCELERATION}

In order to obtain the required minimum quantum lifetime of $0.3 \mathrm{~s}$ for a beam energy of $2.9 \mathrm{GeV}$, the total $\mathrm{rf}$ voltage should be at least $1.7 \mathrm{MV}$. This is most conveniently accomplished with multi-cell cavities which, owing to their large shunt impedance, provide a higher accelerating voltage than single-cell cavities for the same generator power. For the present application, two 500-MHz DORIS-type five-cell cavities from the company Accel have been selected. Each cavity has a shunt impedance of $15 \mathrm{M} \Omega$, enabling both cavities to be driven by a single $75 \mathrm{~kW}$ klystron. A photo of one of the cavities is seen in figure 3 .

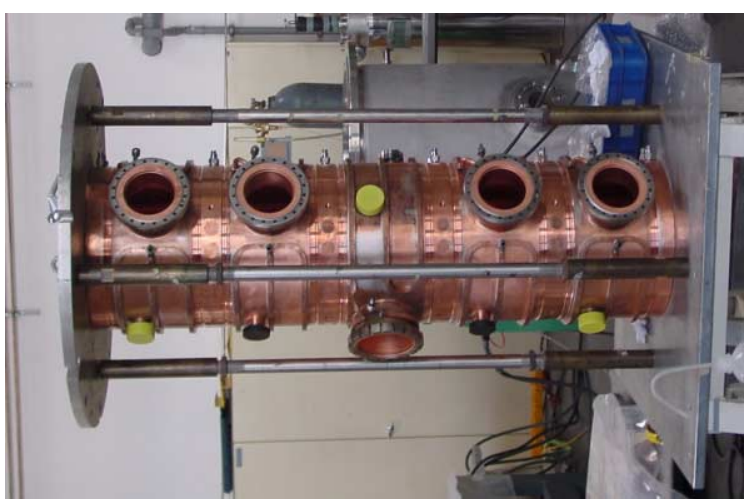

Figure 3: Five-cell cavity (by courtesy of Accel GmbH).

As a result of the finite length of the chopped $500-\mathrm{MHz}$ bunch structure, containing two full or one full plus two halves $2856-\mathrm{MHz}$ bunches, the rf system induces a momentum spread of $\sim 0.6$ percent at injection for an rf voltage of $1.7 \mathrm{MV}$, which is too much considering the maximum horizontal dispersion of $2.8 \mathrm{~m}$. The rf voltage of each five-cell cavity is therefore reduced to $\sim 75 \mathrm{kV}$ at injection which is a good compromise between a large capture efficiency and the increase of the horizontal beam size due to the cavity-induced momentum spread. Unfortunately, the low rf voltage is significantly less than the expected beam induced voltage of $\sim 750 \mathrm{kV}$ (for the maximum linac bunch charge of $\sim 50 \mathrm{pC}$ ) which strongly perturbs the longitudinal dynamics. The problem is reduced considerable by implementing a fast rf feedback loop in the rf system which effectively decreases the shunt impedance of the 
five-cell cavities by a factor $1+\mathrm{G} \sim 10$, where $G$ is the open loop gain of the fast rf feedback loop [3]. In addition, the harmful effect of beam loading is reduced by detuning the five-cell cavities

$$
\Delta \omega=-\frac{R_{s} I_{b}}{2(1+G) Q V_{c a v}} \omega_{c a v}
$$

with respect to the resonance frequency where $R_{s}$ is the shunt impedance of each five-cell cavity, $I_{b}$ is the average beam current, $Q$ is the quality factor of the fivecell cavity, $V_{c a v}$ is the amplitude of the rf voltage of each five-cell cavity, and $\omega_{\text {cav }}$ is the resonant frequency of the five-cell cavity. This particular detuning ensures that the rf voltage is unchanged after the beam of the preinjector is injected into the booster synchrotron, significantly reducing the perturbation of the longitudinal dynamics by transient beam loading. Besides, the detuning reduces the perturbation of the amplitude loop, arising from the rapid beam loading-induced change of the rf voltage at injection which may cause an over-correction of the rf voltage. Operating the rf system with fast rf feedback loop and the optimum detuning, it should be possible to capture and accelerate a large fraction of the injected beam to full energy, facilitating a captured beam current in excess of $10 \mathrm{~mA}$.

During ramping of the beam energy, the dipole magnets are excited by four synchronized arbitrarywaveform power supplies, capable of completing the entire ramping cycle in one second. Similarly, the quadrupole magnets are excited by two synchronized arbitrary-waveform power supplies which can be changed freely with respect to the ramping curve of the dipole magnets, ensuring an accurate control of the transverse tunes throughout the ramping of the beam energy.

The increasing dipole field during ramping gives rise to eddy currents in the dipole magnet vacuum chamber which produces a sextupole field at the position of the beam. It can be shown that the sextupole strength approximately is given by

$$
m=\frac{2}{g} \frac{\mu_{0} \sigma d}{\rho} \frac{d B_{y} / d t}{B_{y}}
$$

where $g$ is the full dipole magnet gap, $\sigma$ is the conductivity of the vacuum chamber material, $d$ is the wall thickness of the vacuum chamber, $\rho$ is the bending radius of the beam trajectory in the dipole magnets, and $B_{y}$ is the vertical component of the bending field [4,5]. For the expected ramping curve of the dipole magnets, the maximum sextupole strength is $\sim 0.17 \mathrm{~m}^{-3}$, equivalent to maximum chromaticity shifts of $\Delta \xi_{x}=3.0$ and $\Delta \xi_{y}=-3.8$ for the horizontal and vertical planes, respectively. Hence, the eddy currents gives rise to a significant modification of the chromaticities with resulting horizontal and vertical chromaticities of $\xi_{x}=-3.8$ and $\xi_{y}=-7.8$, respectively, for the maximum sextupole strength. However, a tracking analysis of the non-linear transverse dynamics reveals that the dynamic aperture is sufficient throughout the whole ramping of the beam energy.

\section{EXTRACTION}

Shortly after the beam has reached the full energy, the electron beam is extracted from the booster synchrotron by positioning the beam at the entrance of the $1.655-\mathrm{m}$ extraction septum magnet by a fast kicker magnet $7.47 \mathrm{~m}$ upstream of the septum magnet. The septum magnet is shielded from the vacuum chamber of the booster synchrotron by a $1.5-\mathrm{mm}$ thick septum sheet placed $18 \mathrm{~mm}$ from the axis of the booster synchrotron. Subsequently, the beam is transported to the extraction transfer line by the septum magnet which deflects the beam $7^{\circ}$ away from the axis of the booster synchrotron in order to clear the downstream quadrupole magnet. The required strength of the kicker magnet is reduced $\sim 30$ percent by locally displacing the beam $9.5 \mathrm{~mm}$ towards the extraction septum sheet during the last $\sim 15 \mathrm{~ms}$ before extraction. The displacement is produced by three bumper magnets near the extraction septum magnet. In order to obtain an extraction efficiency close to 100 percent, the risetime of the kicker magnet is less than $190 \mathrm{~ns}$, facilitating an extraction of the full length of the circulating beam (of $\sim 137 \mathrm{~ns}$ ). For a good capture efficiency of the rf system this will provide an extracted pulse with a beam current above $10 \mathrm{~mA}$.

\section{REFERENCES}

[1] L. Dallin, The Canadian Light Source: An Update, these proceedings.

[2] L. O. Dallin and R. M. Silzer, Booster Ring, http:// www.cls.usask.ca/cls/research/CLS.Design.2.1.28.pdf, Sept. 24, 1999.

[3] A. Gamp, Servo Control of RF Cavities under Beam Loading, in Proc. RF Engineering for Particle Accelerators, CERN Accelerator School, Oxford, 1991, edited by S. Turner (CERN, Geneva, 1992), p. 396.

[4] D. A. Edwards and M. J. Syphers, An Introduction to the Physics of High Energy Accelerators, John Wiley \& Sons, New York, 1993, p. 113.

[5] S. Y. Lee, Nucl. Instr. and Meth. A300, p. 151 (1991). 